\title{
Search for Jovian Hectometric and Kilometric Radiation by GEOTAIL Spacecraft during the Impact of Comet Shoemaker-Levy 9
}

\author{
Yasumasa Kasaba $^{1}$, Hirotsugu KoJima ${ }^{1}$, Hiroshi Matsumoto ${ }^{1}$, and Takeshi Murata ${ }^{2}$ \\ 1 Radio Atmospheric Science Center, Kyoto University, Uji, Kyoto 611, Japan \\ 2 Faculty of Engineering, Ehime University, Bunkyo-cho, Matsuyama 790, Japan
}

(Received August 19, 1995; Revised January 25, 1996; Accepted January 29, 1996)

\begin{abstract}
We made a survey for Jovian hectometric and kilometric radiation by GEOTAIL spacecraft before, during, and after the impacts of Comet Shoemaker-Levy 9 (SL-9). We attempted to identify the radiation from Jupiter based on a $k$ vector determination. However, we did not find clear enhancement of the Jovian radiation activity in the whole period nor around each impact time.

With regard to the Jovian magnetospheric activity, the enhancement of synchrotron and $\mathrm{X}$-ray radiation in association with the SL-9 event has been already reported. Synchrotron radiation increased during the week of the impacts, and X-rays was clearly detected just around $\mathrm{K}$ impact time at the magnetic conjugate footprints of the impact sites. This suggests that a strong perturbation at the impact sites has given non negligible change in the inner magnetosphere directly or indirectly. Our results suggest that such activities were limited in the inner magnetosphere only on low L shells, and there were few amount of direct coupling between the cometary fragments and the Jovian outer magnetosphere. This is consistent with the ground-based observation of the decametric radiation that no significant change was detected during the SL-9 event.
\end{abstract}

\section{Introduction}

The fragments of Comet Shoemaker-Levy 9 (SL-9) collided with Jupiter from July 16 to July 22,1994 . There were predictions that large electric and magnetic turbulence of the Jovian magnetosphere would be created by continuous mass loading (Herbert, 1994), dust-plasma and electrodynamical interactions along the trajectory of the comet (Ip and Prangé, 1994; Bolin and Brenning, 1994; Farrell et al., 1994; de Pater, 1994), and ionized shocks from the impact sites (Kellogg, 1994). These predictions also suggested a long time variation or a short time enhancement of the Jovian non-thermal radio activity. Therefore, observation of these radio activity variations provides us valuable information to study the feature of the magnetospheric turbulence under such extraordinary conditions.

Jovian non-thermal radio emission appears in several wavelength bands; decimetric wavelength (DIM), decametric wavelength (DAM), hectometric wavelength (HOM), and kilometric wavelength (KOM) (cf. Carr et al., 1983; Kaiser, 1989). DIM radiation is synchrotron radiation emitted in the Jovian radiation belt. On the other hand, the DAM/HOM and KOM radiation are thought to be produced by the electron cyclotron maser mechanism and by the linear modeconversion mechanism, respectively. However, precise mechanisms of these emissions are not well understood even today. Therefore, the SL-9 collision events provide new clues for the study of these Jovian radio emissions.

The primary mission objective of the GEOTAIL Plasma Wave Instrument (PWI) (Matsumoto et al., 1994) is to investigate plasma waves and wave-particle interactions in the terrestrial magnetotail. However, it also has a sufficient capability to detect the Jovian HOM and KOM radiation if these radiation are intense enough. In these wavelengths, Ulysses URAP instrument 
(Stone et al., 1992) also made observations on these events. Ulysses was located at the southern solar hemisphere, while GEOTAIL stayed in the ecliptic plane at the whole period of the impacts. Therefore, the two spacecraft had the different view angle toward Jupiter.

We made inquiry into the possible HOM and KOM radiation from Jupiter before, during, and after the period of the impacts. We also compared our results with other observations in various wavelengths.

\section{Observation}

\subsection{Instrumentation}

GEOTAIL was launched on July 24, 1992, and has made extensive observations of various plasma phenomena in the terrestrial magnetotail (e.g. Nishida, 1994).

GEOTAIL Plasma Wave Instrument (PWI) (Matsumoto et al., 1994) is composed of 3 distinct receivers with different frequency and time resolutions: (1) Sweep Frequency Analyzer (SFA) (2) Multi-Channel Analyzer (MCA), and (3) Wave-Form Capture (WFC). The frequency coverages of each receiver are $24 \mathrm{~Hz}-800 \mathrm{kHz}$ (SFA), below $311 \mathrm{kHz}$ (MCA), and below $4 \mathrm{kHz}$ (WFC), respectively. For the observation of the HOM and KOM radiation, we use SFA band-4 (12.5 $\mathrm{kHz}-100 \mathrm{kHz})$ and band-5 $(100 \mathrm{kHz}-800 \mathrm{kHz})$ electric field data. Each receiver has frequency resolution of $1 / 128$ of its frequency bandwidth $(0.684 \mathrm{kHz}$ in band- 4 and $5.47 \mathrm{kHz}$ in band-5), and time resolution of $8 \mathrm{sec}$.

The PWI is connected to two sets of electric dipole antenna systems, a wire dipole antenna (WANT), and a pair of top-hat probe antennas (PANT). SFA was connected to the PANT system during the observation for SL-9 events. The PANT antenna has $100 \mathrm{~m}$ tip to tip length, and was extended perpendicularly to the satellite spin axis. The spin axis is stabilized perpendicular to the ecliptic plane. The spin period is approximately $3 \mathrm{sec}$. Therefore, we can determine the $\mathrm{k}$ vector direction of received waves by monitoring the spin modulation of the wave amplitude.

Figure 1 (a) shows the Jovian location in July 1994 in the heliographic coordinates. Earth is placed in the center of this figure. The distance from Earth to Jupiter was about 5 AU. The Jovian direction was almost perpendicular to that of Sun $\left(102^{\circ}\right.$ in July 11 , and $86^{\circ}$ in July 28). Figure 1 (b) shows the location of GEOTAIL in the Geocentric Solar Ecliptic (GSE) coordinates. GEOTAIL stayed in the distant magnetotail. Figures 1 (a) and (b) indicate that the direction of Jupiter from GEOTAIL was almost perpendicular to that of Sun and Earth, and we could easily distinguish the Jovian radiation from the solar and terrestrial radiation.

\subsection{Observation during the SL-9 impacts period}

Figure 2 (a) is an SFA frequency-time spectrogram, showing the terrestrial radiations from July 11 to 28 , 1994. Below $50 \mathrm{kHz}$, terrestrial continuum radiation was intense. Auroral kilometric radiation (AKR) was very intense from $100 \mathrm{kHz}$ to $500 \mathrm{kHz}$. It is clearly seen that the AKR suffers from a daily frequency modulation. In this spectrogram, the galactic background radiation (Brown, 1973) and instrumental noise are subtracted from data points by standard Fourier techniques (Fainberg, 1979).

Figure 2 (b) shows the AKR index. This index indicates AKR radiation flux normalized to the value at the reference distance $=25 \mathrm{R}_{\mathrm{E}}$, and has a good correlation with magnetic activity (Murata et al., 1995). Thick line indicates the average over 12-hour intervals. In Fig. 2 (a), the lower cutoff frequency of the continuum radiation increases up to above $40 \mathrm{kHz}$ on July 14 . This indicates increase of the solar wind density. Following this density increase, the AKR index was enhanced and continued until July 21. These large geomagnetic disturbances might have given substantial interference on observation of Jovian decametric radio waves on the ground.

To remove contamination from AKR or terrestrial continuum radiation, we analyze the SFA data at $712-800 \mathrm{kHz}$ for the survey of the HOM radiation and at $89-100 \mathrm{kHz}$ for that of the 
(a)

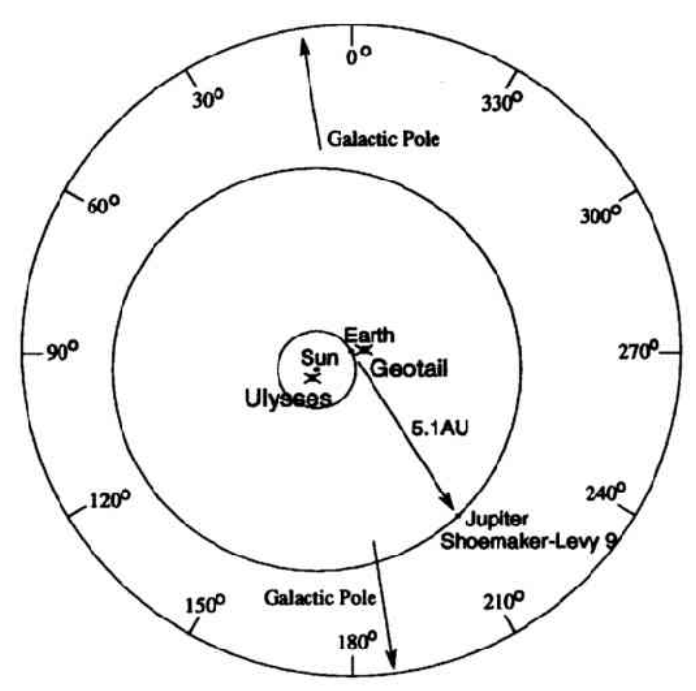

(b)

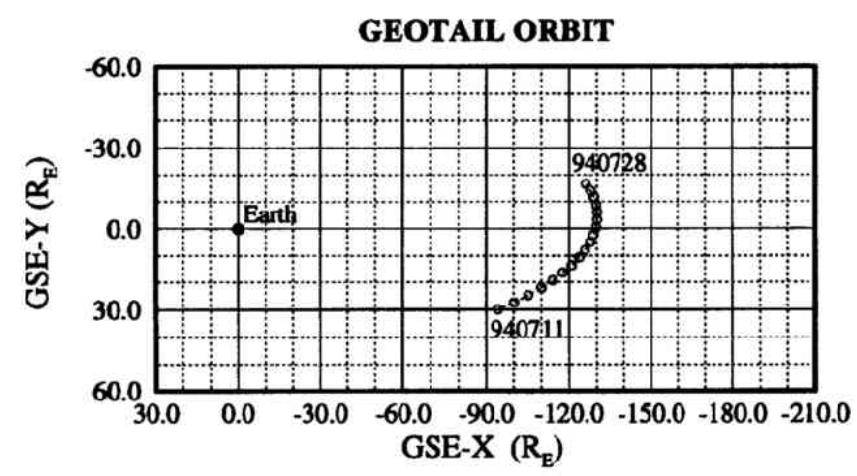

Fig. 1. Location of each object concerned with our observation in July 1994. (a) Location of Sun, Earth, and Jupiter in the heliographic coordinates. Earth is in the center, and the direction of Jupiter from Earth was almost perpendicular to that of Sun $\left(102^{\circ}\right.$ in July 11 , and $86^{\circ}$ in July 28). The location of Ulysses is also shown $\left(-73^{\circ}\right.$ in heliographic latitude). (b) Location of GEOTAIL in the GSE coordinates. GEOTAIL stayed in distant magnetotail in the whole period.

KOM radiation. Figure 3 shows the noise-subtracted radiation flux of (a) $712-800 \mathrm{kHz}$ (average of the 16 frequency components) and (b) $89-100 \mathrm{kHz}$ (average of the 16 frequency components). All the SFA wave data at these two frequency bands were examined at every 1 minute to seek for their propagation direction. The examination was carefully carried out after subtracting the background noise for the whole period mentioned above. Only those with the propagation direction within $\pm 15^{\circ}$ from Jupiter were selected and plotted by solid dots in Fig. 3. Thick lines indicate the average over 12-hour intervals. Horizontal dashed lines show the background noise intensity, which is dominated by the galactic background radiation at $712-800 \mathrm{kHz}$ and instrumental noise at $89-100 \mathrm{kHz}$. From this figure, we could recognize some systematic decrease of the intensities of the HOM and KOM radiation during the week of the impacts. However, both radiation are weaker than background noise level, and we do not remove the possibilities of 
(a)

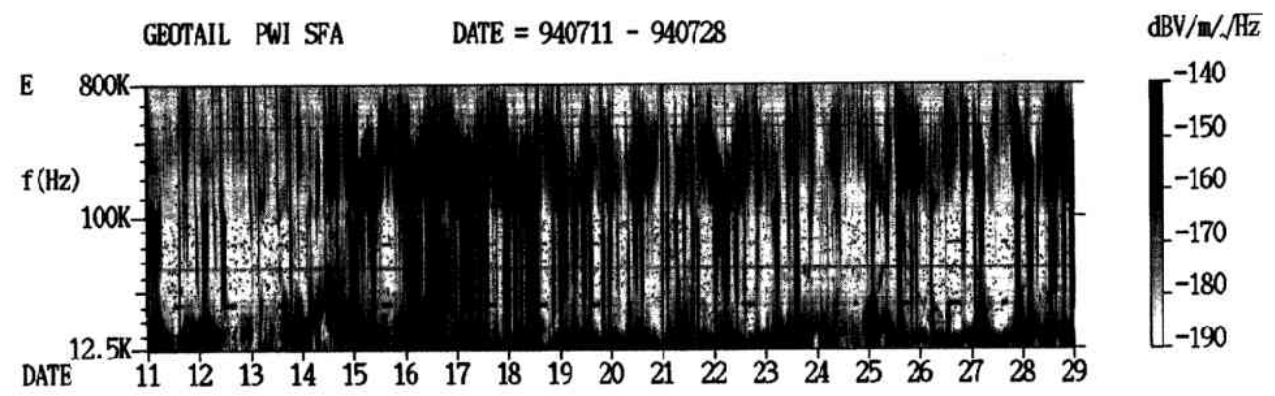

(b)

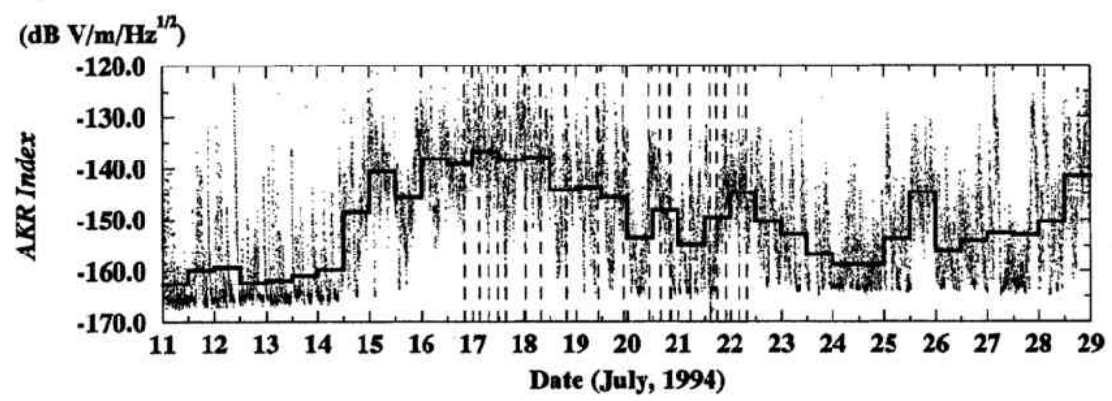

Fig. 2. GEOTAIL PWI/SFA data in July 11-28, 1994. (a) Frequency-Time spectrogram. (b) AKR Index. Thick line shows the average over 12-hour intervals, and dashed vertical line shows the impact time of each fragment : A (20:12UT on July 16), B (2:50UT on July 17), C (7:11UT on July 17), D (11:53UT on July 17), E (15:12UT on July 17), F (0:37UT on July 18), G (7:33UT on July 18), H (19:32UT on July 18), K (10:24UT on July 19), L (22:16UT on July 19), N (10:29UT on July 20), P2 (15:23UT on July 20), Q2 (19:44UT on July 20), Q1 (20:14UT on July 20), R (5:35UT on July 21), S (15:16UT on July 21), T(18:11UT on July 21), U (21:56UT on July 21), V (4:23UT on July 22), W (8:06UT on July 22) (Yeomens and Chodas, private communication, 1995).

contaminations from some natural or artificial effects.

Generally the Jovian HOM and KOM radiation are strongly modulated as a function of the planetary longitude. The HOM radiation has an emission gap around $\mathrm{CML} \sim 200^{\circ}$ (CML : Central Meridian Longitude in System III), and the KOM radiation has a occurrence peak around $\mathrm{CML} \sim 200^{\circ}$. For confirmation of these features, we searched the correlation between the Jovian CML and the radiation flux from the Jovian direction in July 11-16, July 17-22, and July 23-28, 1994 (in Fig. 4). All the data points are the results after the background noise being subtracted. They are selected with the same method as used in Fig. 3. Thick line indicates the average over 30-degree intervals. In the case of the HOM radiation, there seems to be a weak occurrence peak around $\mathrm{CML} \sim 120^{\circ}$ and $\sim 280^{\circ}$, agree with old satellites' results (cf. Lecacheux et al., 1992). On the other hand, there are no systematic modulation in the KOM radiation. This suggests that the Jovian KOM radiation is not clearly detected in our data.

From Fig. 2 and Fig. 3, with exception of the small decrease in the HOM radiation, we can not find clear variation of the Jovian HOM and KOM radiation before, during, and after the impacts period. Jovian radiation in the whole period was weaker than $\sim-180 \mathrm{~dB} \mathrm{~V} \mathrm{~m}^{-1} \mathrm{~Hz}^{-1 / 2}$ 
(a)

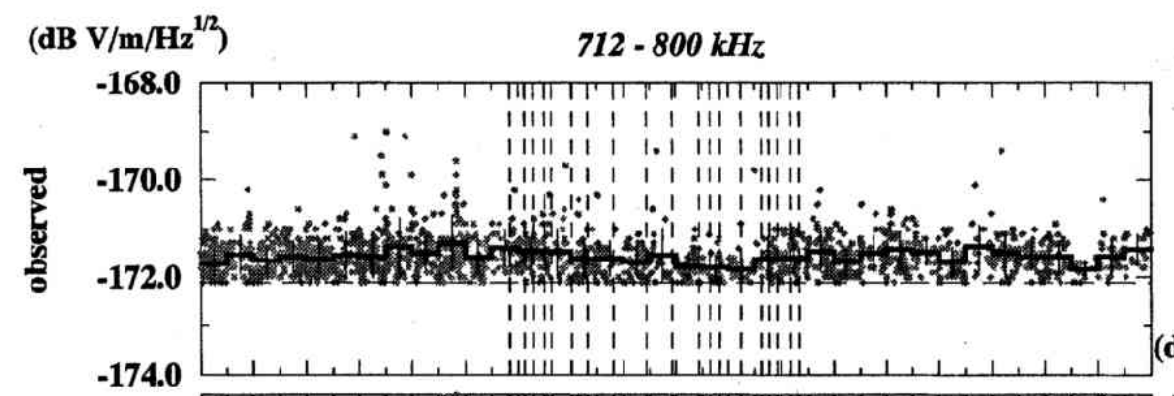

$\left(\mathbf{d B ~ V} / \mathrm{m}^{-\mathrm{Hz}^{1 / 3}}\right)$

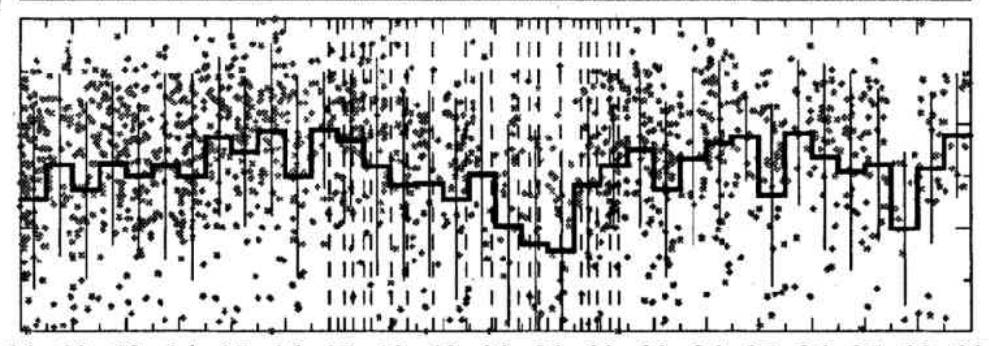

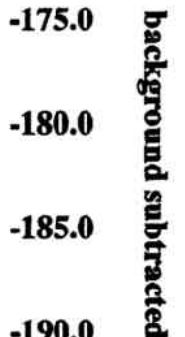

(b)

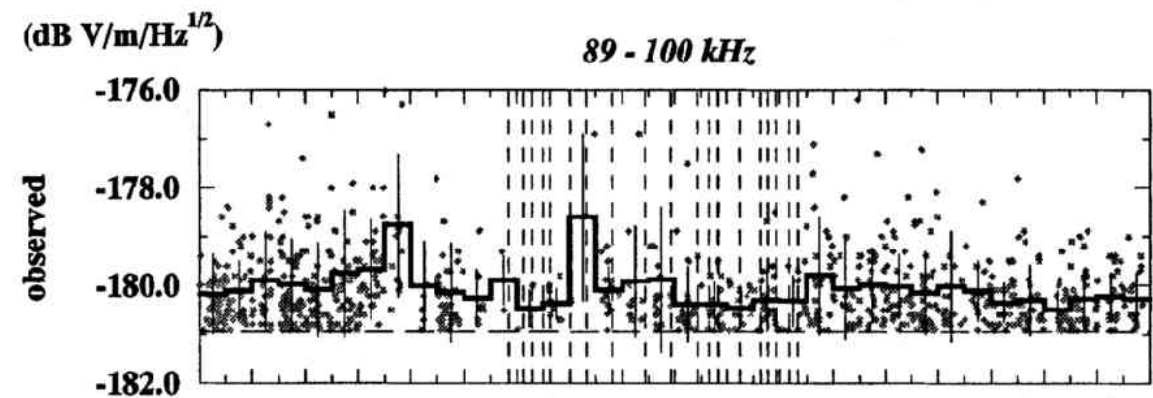

$\left(\mathrm{dB} \mathrm{V} / \mathrm{m} / \mathrm{Hz}^{1 / 2}\right)$

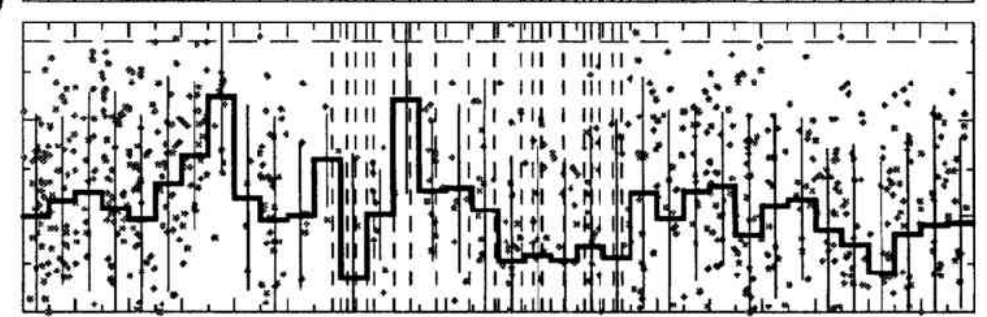

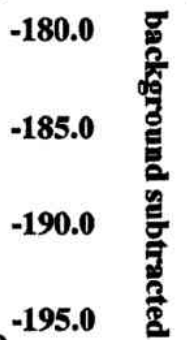

$\begin{array}{llllllllllllllllllll}11 & 12 & 13 & 14 & 15 & 16 & 17 & 18 & 19 & 20 & 21 & 22 & 23 & 24 & 25 & 26 & 27 & 28 & 29\end{array}$

Date (July, 1994)

Fig. 3. Observed radiation fluxes from the Jovian direction in July 11-28, 1994. They are the 1-minute averaged values, which the background noise is subtracted. $Y$-axis is radiation flux $\left(\mathrm{dB} \mathrm{V} \mathrm{m}^{-1} \mathrm{~Hz}^{-1 / 2}\right)$. The $k$ vector directions are determined after background noise subtraction, and only the data which the $k$ vector direction is within $\pm 15^{\circ}$ from the Jovian direction are shown on this figure. Thick line shows the average over 12-hour intervals. $1 \sigma$ value at every 12 hours is overlaid by thin vertical lines. Dashed vertical line shows the impacts time of all fragments. Long dashed horizontal line shows the background noise level. (a) Radiation flux at $712-800 \mathrm{kHz}$ (HOM). Upper panel shows the observed data, and lower panel shows the background subtracted data. (b) Radiation flux at $89-100 \mathrm{kHz}(\mathrm{KOM})$. Upper panel shows the observed data, and lower panel shows the background subtracted data. 
(a)
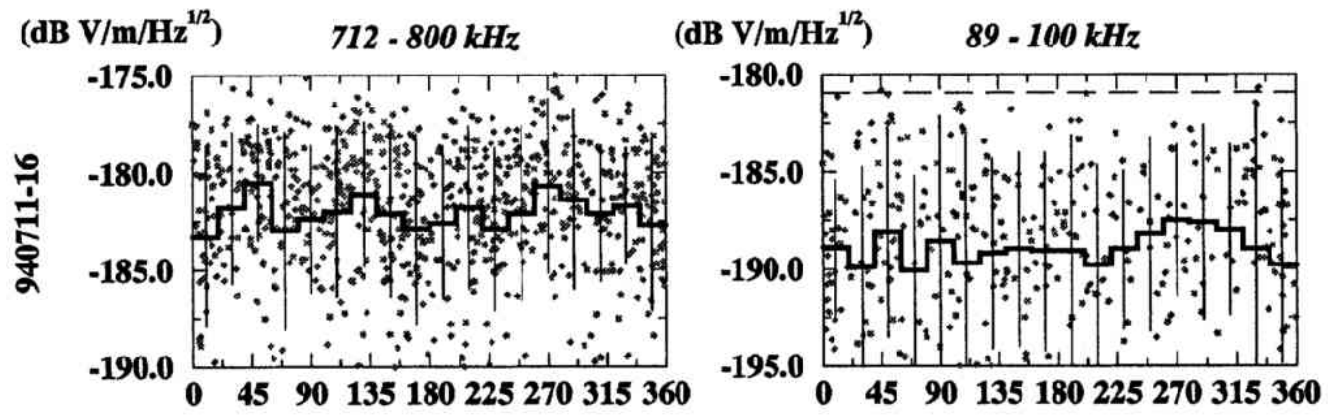

(b)
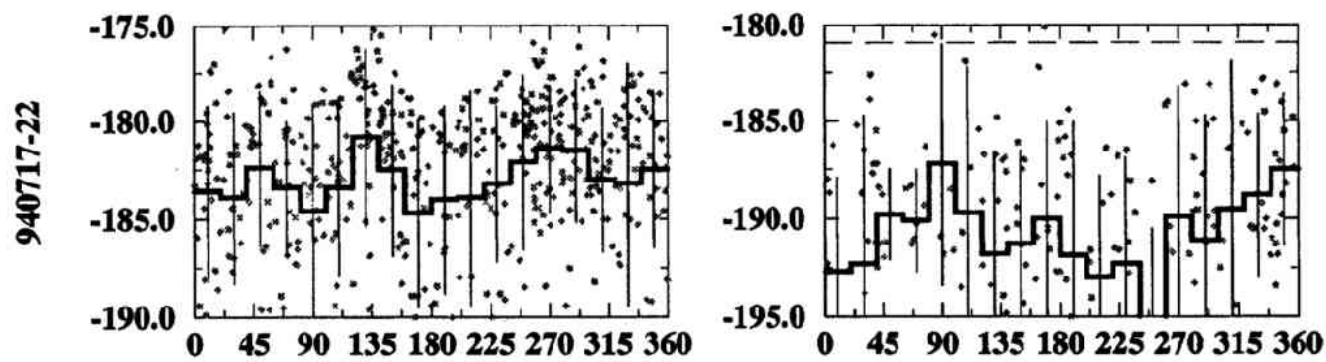

(c)
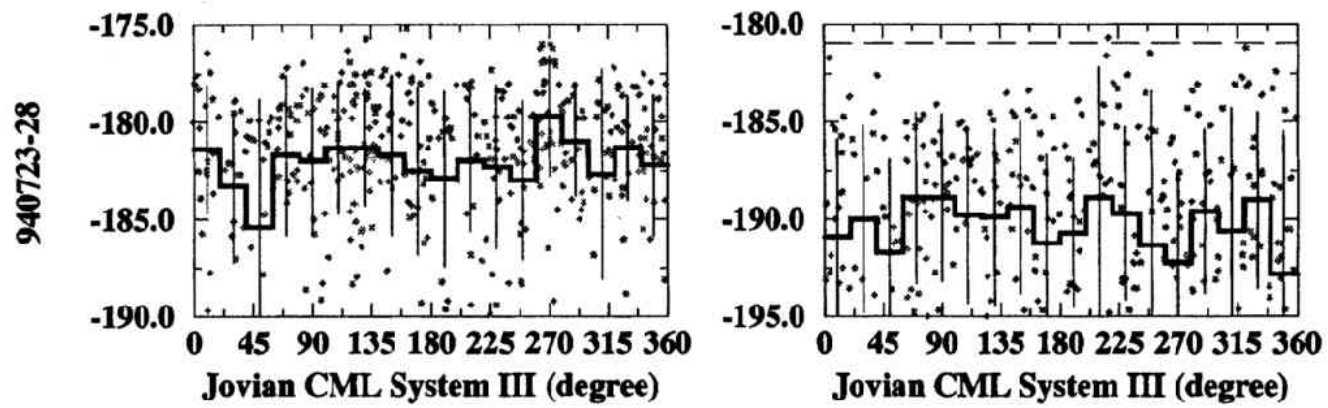

Fig. 4. Correlation between the radiation flux from the Jovian direction and the Jovian CML System III. $Y$-axis is radiation flux $\left(\mathrm{dB} \mathrm{V} \mathrm{m}^{-1} \mathrm{~Hz}^{-1 / 2}\right)$. The data points shows the background subtracted data at 1-minute intervals. Thick line shows the average over $30^{\circ}$ intervals. $1 \sigma$ value at every $30^{\circ}$ is overlaid by a thin vertical line. (a) Correlation on July 11-16, 1994. (b) Correlation on July 17-22, 1994. (c) Correlation on July 23-28, 1994.

$\left(3 \times 10^{-21} \mathrm{~W} \mathrm{~m}^{-2} \mathrm{~Hz}^{-1}\right)$ in the HOM radiation, and $\sim-185 \mathrm{~dB} \mathrm{~V} \mathrm{~m}^{-1} \mathrm{~Hz}^{-1 / 2}\left(1 \times 10^{-21} \mathrm{~W} \mathrm{~m}^{-2}\right.$ $\mathrm{Hz}^{-1}$ ) in the KOM radiation. This is consistent with the Jovian radiation flux level in its normal activity, $\sim 10^{-21} \mathrm{~W} \mathrm{~m}^{-2} \mathrm{~Hz}^{-1}$ [calculated from Ulysses observation (cf. Reiner et al., 1993)].

Ulysses also made observation during this period (Desch et al., 1995). They did not get enhancement in the HOM radiation, either. On the other hand, they observed $15 \mathrm{~dB}$ enhancement of the KOM radiation during the $\mathrm{P} / \mathrm{Q}$ fragment impacts on July 20. However, we detected strong AKR enhancement coincidently and could not confirm this event. (They conclude this 
as "association with unusual high pressure solar wind streams", and not linked with the impact events.)

\subsection{Observation with each impact time}

Around the K impact time, ROSAT detected a large X-ray enhancement (Waite et al., 1995). Figure 5 (a) shows the frequency-time diagram around the K impact time (10:24UT, July 19). There were very clear enhancement at about $800 \mathrm{kHz}$ immediately after the $\mathrm{K}$ impact time. Figure 5 (b) shows the SFA flux-time diagram around the K impact time. Small and large dots indicate the radiation flux from the omni-direction and from the Jovian direction, respectively. In this figure, the enhancement in Fig. 5 (a) is also shown in the radiation from omni-direction, but not shown in the radiation from the Jovian direction. This enhancement, however, turns out to be a solar type III radio burst by the $k$ vector direction.

Figure 5 (c) shows the flux-time diagram around the impact times of six large fragments $(G$, H, K, L, Q1, and S). From Figs. 5 (b) and (c), radiation flux from the Jovian direction stayed almost at same level during the whole period. The HOM radiation from the Jovian direction was distinguished more frequently at $0.5 \sim 1.5$ hours before the time of each impact event. This suggests the existence of the precursor radiation. However, we have no confidence about this conclusion because it was not accompanied by any clear flux enhancement.

\section{Discussion}

We did not identify a clear enhancement of the HOM or KOM radiation activity related to the impacts, except for small variation in the HOM radiation activity. For enhancement of the Jovian HOM and KOM radiation, large electromagnetic turbulence would be needed in their source locations or on the magnetic field lines penetrating through their generation region. The source location of the HOM radiation is at $\sim 3 \mathrm{R}_{\mathrm{J}}$ from the planet, and on the $L \sim 4$ to 6 shells (Reiner et al., 1993), the source location of the KOM radiation is thought to originate in the Io torus or above the polar ionosphere (cf. Kaiser, 1989). Our data suggest that there were few turbulence in these locations.

By ground-based Jovian radio wave observation, the enhancement of the DIM synchrotron radiation was reported during the week of impacts (cf. Dulk et al., 1995; Leblanc and Dulk, 1995; Klein et al., 1995; Bolton et al., 1995). The DIM radiation occurred near the Jovian magnetic equator at $\sim 1-2 R_{J}$ from the planet, and generated by relativistic electrons trapped in the Jovian magnetic field (cf. Kaiser, 1989). This observation suggests that population of energetic electrons in the Jovian radiation belt increased in this term. On the other hand, ROSAT detected soft $\mathrm{X}$-ray enhancement at the magnetic conjugate footprints of impact site just around $\mathrm{K}$ impact time (Waite et al., 1995). It seems that bremsstrahlung radiation is produced by energetic electrons, which were accelerated at the impact site or precipitated from the radiation belt after being scattered with the ionized shock (cf. Kellogg, 1994). These processes also account for the enhancement of synchrotron radiation through electron heating in the radiation belt. The impact sites are distributed on low $L$ shells $(L \sim 1.5$ to 3 ). Therefore we conclude that these turbulence were caused at or near these impact sites, and not affected the outer magnetosphere on large $\mathrm{L}$ shells.

There were another possible turbulence created by the passage of cometary fragments in the Jovian outer magnetosphere: increase of the charged particle population by trapping of plasma gas and charged dusts (Herbert, 1994), field-aligned current (Ip and Prangé, 1994), and generation of field-aligned current by dust-plasma or electrodynamic interaction (Bolin and Brenning, 1994; Farrell et al., 1994). The first may generate the activity increase for several weeks or months after the impact period, and the second and the third may provide the precursor radiation before the impact time of each fragment. Except for weak precursor feature of the HOM radiation, we find 
(a)

GEOTAIL PWI SFA DATE $=940719$

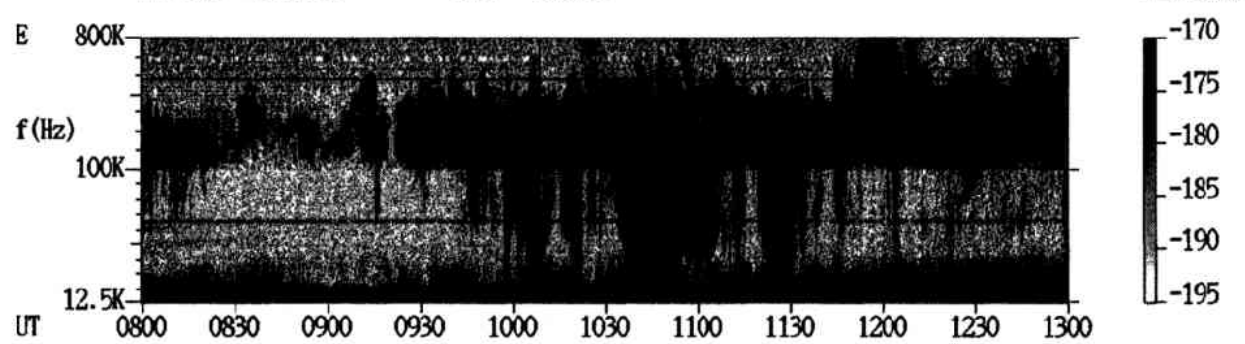

(b)
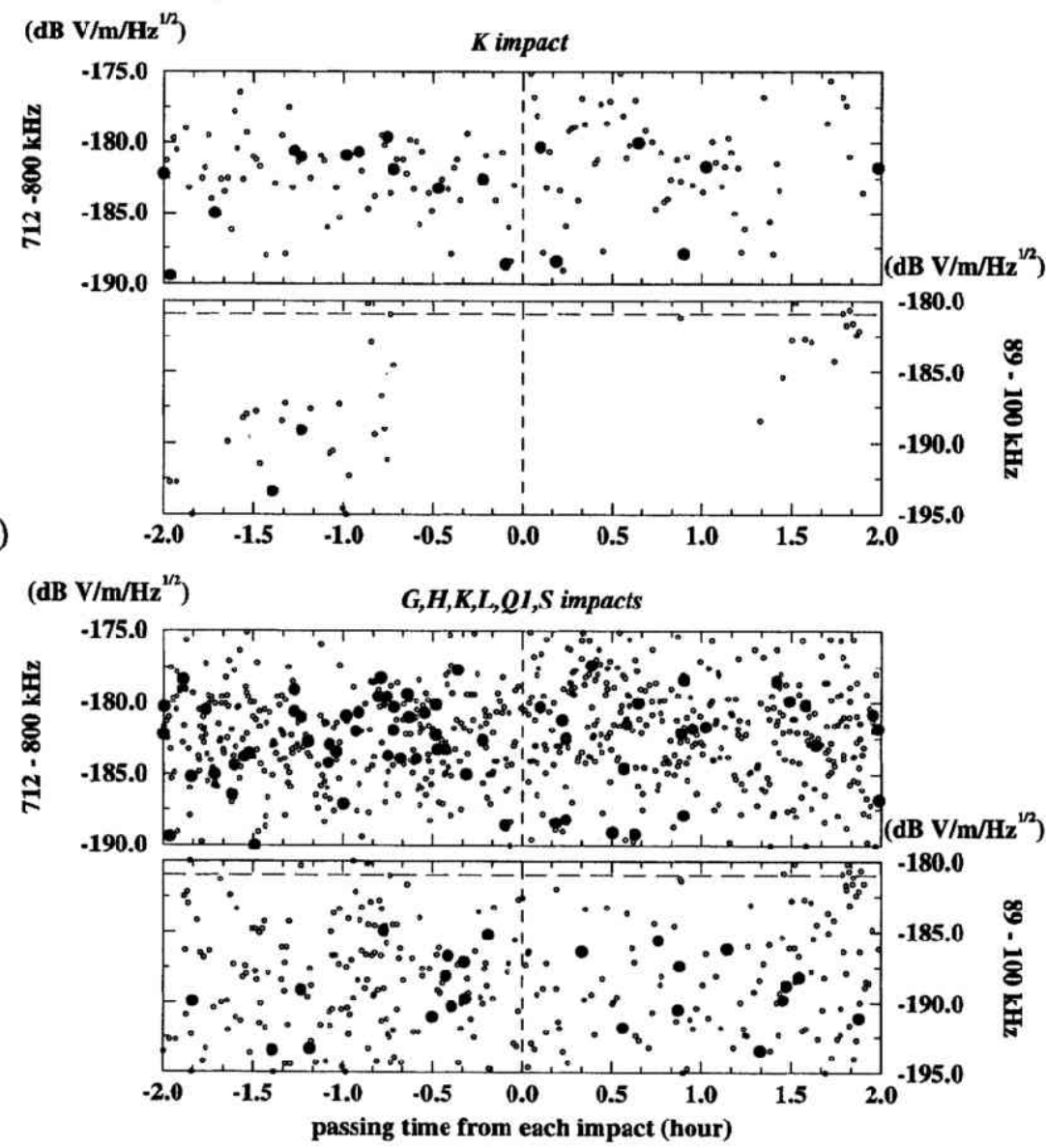

Fig. 5. Radiation flux from the Jovian direction around the each impact time. (a) Frequency-Time spectrogram of 8-13UT on July 19, around the K impact time (10:24UT). Two faint enhancement around $800 \mathrm{kHz}$ (10:26UT, 10:55UT) are solar type III radio bursts. (b) Radiation flux from the Jovian direction around the K impact time. $Y$-axis is radiation flux $\left(\mathrm{dB} \mathrm{V} \mathrm{m}^{-1} \mathrm{~Hz}^{-1 / 2}\right)$. Upper and lower panels show the data at $712800 \mathrm{kHz}$ and $89-100 \mathrm{kHz}$, respectively. Small and large circles show the data from the omni-direction and from the Jovian direction, respectively. Dashed vertical line shows the K impact time. Long dashed horizontal line shows the background noise level. (c) Radiation flux around G, H, K, L, Q1, and S impact times. Other features are as same as in Fig. 5 (b). 
no clear variation of radio activity before and after the each impact time. This suggests that there are few amount of such direct interactions between the comet and Jovian outer magnetosphere. In the Jovian magnetosphere, Io is always running accompanied by the plasma torus, and we can also conclude that the effect from the passage of cometary fragments seems to be weaker than from Io. In addition, by satellite observations with HST, IUE, and EUVE spacecraft, and by ground-based radio observations, the Io plasma torus has not been significantly affected by either the comet fragments' passage through the magnetosphere or impact with the planet (cf. McGrath et al., 1995; Brown et al., 1995). The Io plasma torus is one of the source regions of the KOM radiation, and this is consistent with our results.

On the other hand, Carr et al. (1995) concluded from ground-based monitoring observations that there was no significant change in the Jovian decametric emission activity before, during, and after the impact period. This is consistent with our results, "The turbulence in the Jovian outer magnetosphere was weak.", because the DAM and HOM radiation are thought to be produced by the same cyclotron maser mechanism at different altitudes in the Jovian magnetosphere. However, it is noted that the emission features of the DAM and HOM radiation are generally different. For example, the DAM radiation is controlled by Io location strongly, but the HOM radiation is not.

\section{Conclusions}

GEOTAIL detected no clear enhancement of the Jovian non-thermal HOM and KOM radiation in the whole impacts period. We find no change in features of the KOM radiation activity. In the HOM radiation, however, a small decrease of the activity during the week of impacts as well as small enhancement of the precursor activity accompanied with each impact. But these features are weaker than the galactic background radiation, and we could not show a confident evidence. Jovian radiation during this period was weaker than $\sim-180 \mathrm{~dB} \mathrm{~V} \mathrm{~m}^{-1} \mathrm{~Hz}^{-1 / 2}$ in the HOM radiation, and $\sim-185 \mathrm{~dB} \mathrm{~V} \mathrm{~m}^{-1} \mathrm{~Hz}^{-1 / 2}$ in the KOM radiation.

X-ray radiation enhancement (Waite et al., 1995) and synchrotron radiation enhancement (cf. Dulk et al., 1995; Leblanc and Dulk, 1995; Klein et al., 1995; Bolton et al., 1995) suggests the large turbulence in the Jovian inner magnetosphere. However, there was no clear activity variation in the Jovian outer magnetosphere. We conclude that there were few effects from mass loading or direct interactions between the cometary fragments and Jovian outer magnetosphere along the comet trajectory. This conclusion is consistent with no significant change of the DAM radiation monitored on the ground (cf. Carr et al., 1995).

We would like to express our sincere thanks to GEOTAIL team for the preparation and successful operation of this special observation. We also express our thanks to S. Chikuba, H. Hamada, S. Horiyama, J. Koizumi, T. Miyake for their contributions to intensive GEOTAIL PWI data analysis for the SL-9 impact period. Y. K. would like to express thanks to J. H. Waite Jr. for valuable information on ROSAT observation, to R. J. MacDowall, M. L. Kaiser, M. J. Reiner, and M. D. Desch for valuable information on Ulysses observation and Jovian radio emission. We would like to express our thanks to K. Hashimoto, I. Nagano, and T. Terasawa for their fruitful discussion.

\section{REFERENCES}

Bolin, O. and N. Brenning, Electrodynamical interaction between comet Shoemaker-Levy 9 and Jupiter, Geophys. Res. Lett., 21, 1063-1066, 1994.

Bolton, S. J., R. S. Foster, and W. B. Waltman, Observations of Jupiter's synchrotron radiation at $18 \mathrm{~cm}$ during the Comet Shoemaker-Levy 9 impacts, of Comet Shoemaker-Levy 9, Geophys. Res. Lett., 22, 1801-1804, 1995.

Brown, L. W., The galactic radio spectrum between 130 and $2600 \mathrm{kHz}$, Astrophys. J., 180, 359-370, 1973 .

Brown, M. E., E. J. Moyer, A. H. Bouchez, and H. Spinrad, Comet Shoemaker-Levy 9: No effect on the Io plasma torus, Geophys. Res. Lett., 22, 1833-1836, 1995. 
Carr, T. D., M. D. Desch, and J. K. Alexander, Phenomenology of magnetospheric radio emissions, in Jupiter, edited by E. J. Desseler, 226-284, Cambridge University Press, New York, 1983.

Carr, T. D., F. Reyes, J. A. Phillips, J. May, L. Wang, J. Aparici, H. Alvarez, F. Olmos, L. Garcia, J. M. De Buizer, W. B. Greenman, T. Clark, J. Levy, S. Padin, and C. A. Higgins, Results of decametric monitoring of the comet collision with Jupiter, Geophys. Res. Lett., 22, 1785-1788, 1995.

de Pater, I., The effect of comet Shoemaker-Levy 9 on Jupiter's synchrotron radiation, Geophys. Res. Lett., 21, 1071-1074, 1994.

Desch, M. D., M. L. Kaiser, W. M. Farrell, R. J. MacDowall, and R. G. Stone, Traversal of comet SL-9 through the Jovian magnetosphere and impact with Jupiter: Radio upper limits, Geophys. Res. Lett., 22, 1781-1784, 1995.

Dulk, G. A., Y. Leblanc, and R. W. Hunstead, Flux and images of Jupiter at 13, 2, and $36 \mathrm{~cm}$ before, during, and after SL9 impacts, Geophys. Res. Lett., 22, 1789-1792, 1995.

Fainberg, J., Technique to determine location of radio sources from measurements taken on spinning spacecraft, NASA Technical Memorandum, 80598, 1979.

Farrell, W. M., M. L. Kaiser, M. D. Desch, and R. J. MacDowall, Possible radio wave precursors associated with the comet Shoemaker-Levy 9/Jupiter impacts, Geophys. Res. Lett., 21, 1067-1070, 1994.

Ip, W.-H. and R. Prangé, On possible magnetospheric dust interactions of comet Shoemaker-Levy 9 at Jupiter, Geophys. Res. Lett., 21, 1051-1051, 1994.

Herbert, F., The impact of Comet Shoemaker-Levy 9 on the Jovian magnetosphere, Geophys. Res. Lett., 21, 1047-1050, 1994.

Kaiser, M. L., Observations of non-thermal radiation from planets, in Plasma Waves and Instabilities at Comets and in Magnetospheres, edited by B. T. Tsurutani and H. Oya, Geophys. Monogr., 53, AGU, 221-237, Washington, D. C., 1989.

Kellogg, P. J., Plasma effects on the interaction of a comet with Jupiter, Geophys. Res. Lett., 21, 1055-1058, 1994.

Klein, M. J., S. Gulkis, and S. J. Bolton, Changes in Jupiter's 13-cm synchrotron radio emission following the impact of Comet Shoemaker-Levy 9, Geophys. Res. Lett., 22, 1797-1800, 1995.

Leblanc, Y. and G. A. Dulk, Changes in brightness of Jupiter's radiation belts at 13 and $22 \mathrm{~cm}$ during and after impacts of Comet SL9, Geophys. Res. Lett., 22, 1793-1796, 1995.

Lecacheux, A., B. M. Pedersen, Ph. Zarka, M. G. Aubier, M. D. Desch, W. M. Farrell, M. L. Kaiser, R. J. MacDowall, and R. G. Stone, In ecliptic observations of Jovian radio emissions by Ulysses comparison with Voyager results, Geophys. Res. Lett., 19, 1307-1310, 1992.

Matsumoto, H., I. Nagano, R. R. Anderson, H. Kojima, K. Hashimoto, M. Tsutsui, T. Okada, I. Kimura, Y. Omura, and M. Okada, Plasma wave observations with GEOTAIL spacecraft, J. Geomag. Geoelectr., 46 , 59-95, 1994.

McGrath, P. J., D. T. Hall, P. L. Matheson, H. A. Weaver, J. T. Trauger, T. E. Smith, N. Thomas, R. Glabstone, N. M. Schneider, W. M. Harris, T. A. Livengood, R. Prangé, and M. C. Festou, Response of the Io plasma torus to Comet Shoemaker-Levy 9, Science, 267, 1313-1317, 1995.

Murata, T., H. Matsumoto, H. Kojima, T. Iyemori, T. Nagai, and Y. Kasaba, Proposal of a New Index for Substorm Study: AKR Index, J. Geophys. Res., 1995 (to be submitted).

Nishida, A., The GEOTAIL mission, Geophys. Res. Lett., 21, 2871-2874, 1994.

Reiner, M. J., J. Fainberg, and R. G. Stone, Source characteristics of Jovian hectometric radio emissions, J. Geophys. Res., 98, E10, 18767-18777, 1993.

Stone, R. G., J. L. Bougeret, J. Caldwell, P. Canu, Y. de Conchy, N. Cornilleau-Wehrlin, M. D. Desch, J. Fainberg, K. Goetz, M. L. Goldstein, C. C. Harvey, S. Hoang, R. Howard, M. L. Kaiser, P. J. Kellogg, B. Klein, R. Knoll, A. Lecacheux, D. Lengyel-Frey, R.J. MacDowall, R. Manning, C. A. Meetre, A. Meyer, N. Monge, S. Monson, G. Nicol, M. J. Reiner, J. L. Steinberg, E. Torres, C. de Villedary, F. Wouters, and P. Zarka, The unified radio and plasma wave investigation, Astron. Astrophys. Suppl. Ser., 92, 291-316, 1992.

Waite, Jr., J. H., G. R. Gladstone, K. Franke, W. S. Lewis, A. C. Fabian, W. N. Brandt, C. Na, F. Haberl, J. T. Clarke, K. C. Hurley, M. Sommer, and S. Bolton, ROSAT observations of X ray emissions from Jupiter during the impact of Comet Shoemaker-Levy 9, Science, 268, 1598-1601, 1995. 